Short report

\title{
The significance of the incidental finding of basal ganglia calcification on computed tomography
}

\author{
MG HARRINGTON, P MACPHERSON, WB MCINTOSH,* BF ALLAM,* I BONE \\ From the Institute of Neurological Sciences, Southern General Hospital, and the Department of \\ Biochemistry, Stobhill General Hospital,* Glasgow
}

SUMMARY Basal ganglia calcification was found as an incidental finding in 42 out of 7000 patients who underwent computed tomography. The calcification showed on plain skull radiography when the maximum density on computed tomography exceeded 100 Hounsfield units. The 26 patients with basal ganglia calcification detected on computed tomography who were available for follow-up, were investigated with matched controls. No clinical features of basal ganglia calcification were noted. Twenty-four patients had no significant metabolic abnormality and two patients had parathyroid disorder identified.

Basal ganglia calcification identified radiographically has been associated with any one of 24 conditions $^{12}$ (table) with treatable parathyroid disease being the most common of these associations. ${ }^{3}$ Computed tomography (CT) has identified basal ganglia calcification more sensitively than plain skull radiographs, ${ }^{2-9}$ but the incidence of disease including parathyroid disorder in the CT group has been less. ${ }^{24-7}$ This study was carried out to correlate radiographically basal ganglia calcification on CT with that on plain skull radiographs; to attempt to define any systemic metabolic mechanism of basal ganglia calcification formation; and to investigate the possibility of early detection of treatable parathyroid disease.

Address for reprint requests: Dr MG Harrington, The Institute of Neurological Sciences, Southern General Hospital, Glasgow G51 4TF, Scotland.

Received 16 July 1981 and in revised form 12 September 1981. Accepted 26 September 1981

\section{Patients and methods}

The CT scans of patients who had attended the Institute of Neurological Sciences from 1975 to 1980 were reviewed for evidence of basal ganglia calcification. From approximately 7000 patients, 15778 scans were obtained and 42 cases of basal ganglia calcification were found. Sixteen had died or were lost to follow-up. The 26 remaining patients were matched for age, sex, clinical diagnosis and drug treatment with controls. All patients and controls had the following investigations: full clinical assessment; a lateral, a $20^{\circ} \mathrm{AP}$, and Towne view skull radiograph were taken on a Siemens CRT 7 using the $\mathbf{0 . 3} \mathrm{mm}$ focal spot. CT was performed on an EMI head scanner using a $160 \times$ 160 matrix (except for the first $1 \frac{1}{2}$ years when the matrix was $80 \times 80$ ).

The presence or absence of relevant calcification was recorded, its distribution noted and the average and maximum density in Hounsfield units $(\mathbf{H})$ was determined for each region of calcification. Venous blood was taken without haemostasis and casual urine samples were obtained from fasting, rested patients. Serum electrolytes urea, creatinine, calcium, phosphate, total protein

Table 24 conditions described ${ }^{12}$ in association with radiographically identified basal ganglia calcification

Idiopathic hypoparathyroidism

Secondary hypoparathyroidism

Pseudohypoparathyroidism

Pseudo-pseudohypoparathyroidism

Hyperparathyroidism

Hypothyroidism

Birth anoxia

Carbon monoxide intoxication

Lead intoxication

Fahr's syndrome (ferrocalcinosis)

Familial idiopathic symmetrical basal ganglia calcification

Hastings-James syndrome (idiopathic lenticulo-dentate calcification)
Cockayne's syndrome

Lipoid proteinosis (hyalinosis cutis)

Tuberose sclerosis

Parkinsonism

Vascular disease

Cerebral haemorrhage

Radiation therapy

Methotrexate therapy

Cytomegalic inclusion disease

Encephalitis (measles, chicken pox)

Toxoplasmosis

Cysticercosis 
albumin, bilirubin, alkaline phosphatase, transaminases, gamma GT, creatine kinase, urates, cholesterol and magnesium were measured by standard techniques as were urinary electrolytes, urea, creatinine, calcium, phosphate and magnesium. Serum calcium was corrected for albumin, ${ }^{10}$ urine calcium/creatinine ratio and renal threshold phosphate concentration $\left(\mathrm{TmPO}_{4} / \mathrm{GFR}\right)^{11}$ were derived.

\section{Results}

The diagnosis of the patient group was most frequently epilepsy, headache, cerebral infarction or dementia. No clinical evidence of basal ganglia disease was found in the patient group. Features of idiopathic hypoparathyroidism and pseudohypoparathyroidism were each found in one patient. Of the eight epileptic patients, one had cataracts, macular degeneration, sensori-neural dearness, psychoneurosis and hypertension with negative family history; another two had had birth anoxia. One male with optic atrophy had low intelligence. All but nine patients were receiving drugs: most commonly these were anticonvulsants, diuretics or steroids.

Plain skull radiographs showed basal ganglia calcification in eight out of 26 patients. There was no correlation between the distribution of basal ganglia calcification on CT and underlying disease. Fifteen patients had more or less identical density of basal ganglia calcification in each hemisphere; two more were significantly denser in the right hemisphere and seven more were denser in the left hemisphere (a significant difference being greater than $20 \mathrm{H}$ ). In general basal ganglia calcification showed on the plain skull radiographs where the maximum density on CT was greater than $100 \mathrm{H}$ and where average density was greater than $65 \mathrm{H}$. The only exception was one patient who had a maximum density of $211 \mathrm{H}$ and average density of $71 \mathrm{H}$ with negative plain skull radiographs; this was explained by the very small area of calcification involved.

There was no significant difference between the patient and control groups in any of the direct and derived biochemistry, except for the serum albumin, where the control group had a lower range than the patient group $(p<0.002)$. but both were within the normal range. Of all other indices, the following were normal: serum electrolytes, urea, creatinine, urate, cholesterol, bilirubin, transaminases and lactate dehydrogenase. Elevated blood glucose was present in five patients with either idiopathic or steroidinduced diabetes. Elevated serum gamma GT was found in five patients who were either on anticonvulsant therapy or were regular alcohol drinkers and the same patients had elevated serum alkaline phosphatase levels (the increased isoenzyme being of liver origin.) One patient had biochemistry typical of idiopathic hypoparathyroidism and one of pseudohypoparathyroidism. There were isolated mild abnormalities of either serum phosphate, magnesium $\mathrm{TmPO}_{4} / \mathrm{GFR}$ or urine calcium/creatinine ratio in nine patients not suggestive of significant metabolic disease.

\section{Discussion}

Anticonvulsant therapy has been linked with basal ganglia calcification ${ }^{4}$; however, there is no evidence that anticonvulsant therapy per se causes such calcification. Of our eight epileptic patients, all taking anticonvulsant therapy, two had confirmed parathyroid disorder and a further three had isolated biochemical abnormalities of doubtful significance. Our control group also received anticonvulsant therapy. Osteomalacia has occasionally been described with anticonvulsant therapy but it is not known to be associated with basal ganglia calcification.

Radiologically, where basal ganglia calcification has been identified by $\mathrm{CT}$, the rate of detection by plain skull radiographs ranges from $7-10 \% \cdot{ }^{56}{ }^{9}$ Our higher proportion of $30 \%$ is probably attributable to the fine focus $x$-ray tube used. It has been stated ${ }^{2}$ that where regions other than the globus pallidus were involved, "pathological calcification" existed. We accept that this is frequently the case where metabolic groups are being studied but we did not find this correlation. Indeed our patient with idiopathic hypoparathyroidism had negative plain films and mild calcification of only the globus pallidus, while one of the most florid examples of basal ganglia calcifications on $\mathrm{CT}$ with positive plain skull radiographs was a 43-year-old man with headaches, no drug therapy and normal clinical and biochemical assessment. Owing to the superior sensitivity of CT over plain skull radiographs in the detection of basal ganglia calcification, the patient with idiopathic hypoparathyroidism was undoubtedly diagnosed earlier.

\section{References}

${ }^{1}$ Reader MM, Felson B. In: Reader MM, Felson B, eds. Gamuts of Radiology. Oxford: Pergamon Press. 1975 : A40.

${ }^{2}$ Cohen CR, Duchesnau PM, Weinstein MA. Calcification of the basal ganglia as visualised by computed tomography. Radiology 1980;134:97-9.

${ }^{3}$ Sutton D. In: Sutton D, ed. A textbook of radiology and imaging. 3rd ed. Edinburgh: Churchill Livingstone, 1980:1101.

${ }^{4}$ Brannan TS, Burger AA, Chaudhury MY. Bilateral basal ganglia calcifications visualised on CT scan. 
J Neurol Neurosurg Psychiatry 1980;43:403-6.

${ }^{5}$ Koller WC, Cochran JW, Klawans HL. Calcification of the basal ganglia: computerised tomography and clinical correlation. Neurology (Minneap) 1979;29: 328-33.

${ }^{6}$ Murphy MJ. Clinical correlations of CT scan detected calcification of the basal ganglia. Ann Neurol 1979;6: 507-11.

'Sachs C, Ericson K, Erasmie U, Bergstrom M. Incidence of basal ganglia calcifications on computed tomography. J Comput Assist Tomogr 1979;3:339-44.

${ }^{8}$ Smith D, Bloch S, Al-Rashid RA. Basal ganglia calcifications on CT scanning in children with acute lymphocytic leukaemia. Neuroradiology 1980;20:913.

${ }^{9}$ Danzigger A, Talk W, Sandler M, Forman M. Computed tomography in basal ganglia calcification. Clin Radiol $1980 ; 31: 167-8$.

${ }^{10}$ Imrie CW, Allam BF, Ferguson JC. Hypocalcaemia of acute pancreatitis: the effect of hypoalbuminaemia. Cur Med Res Opin 1976;4:101-16.

${ }^{11}$ Walton RJ, Bijvoet OLM. Nomogram for derivation of renal threshold phosphate concentration. Lancet 1975 ;(ii):309-10 